\title{
Real-time monitoring of tumor vascular disruption induced by radiofrequency assisted gadofullerene
}

\author{
Ruijun Deng ${ }^{1,2 \dagger}$, Yuqing Wang ${ }^{3 \dagger}$, Mingming Zhen ${ }^{1,2^{*}}$, Xue $\mathrm{Li}^{1,2}$, Toujun Zou ${ }^{1,2}$, Jie Li ${ }^{1,2}$, Tong Yu ${ }^{1,2}$, \\ Yue Zhou ${ }^{1,2}$, Zhigao Lu ${ }^{1,2}$, Hui Xu ${ }^{1}$, Chunying Shu ${ }^{1,2}$ and Chunru Wang ${ }^{1,2^{*}}$
}

\begin{abstract}
The anti-vascular therapy has been extensively studied for high performance tumor therapy by suppressing the tumor angiogenesis or cutting off the existing tumor vasculature. We have previously reported a novel anti-tumor treatment technique using radiofrequency (RF)-assisted gadofullerene nanocrystals (GFNCs) to selectively disrupt the tumor vasculature. In this work, we further revealed the changes on morphology and functionality of the tumor vasculature during the high-performance RF-assisted GFNCs treatment in vivo. Here, a clearly evident mechanism of this technique in tumor vascular disruption was elucidated. Based on the $\mathrm{H} 22$ tumor bearing mice with dorsal skin flap chamber (DSFC) model and the dynamic contrast enhanced magnetic resonance imaging (DCE-MRI) technique, it was revealed that the GFNCs would selectively inset in the gaps of tumor vasculature due to the innately incomplete structures and unique microenvironment of tumor vasculature, and they damaged the surrounding endothelia cells excited by the RF to induce a phase transition accompanying with size expansion. Soon afterwards, the blood flow of the tumor blood vessels was permanently shut off, causing the entire tumor vascular network to collapse within $24 \mathrm{~h}$ after the treatment. The RF-assistant GFNCs technique was proved to aim at the tumor vasculature precisely, and was harmless to the normal vasculature. The current studies provide a rational explanation on the high efficiency anticancer activity of the RF-assisted GFNCs treatment, suggesting a novel technique with potent clinical application.
\end{abstract}

Keywords: gadofullerene, radiofrequency, dorsal skin flap chamber, dynamic contrast enhanced magnetic resonance imaging, tumor vasculature

\section{INTRODUCTION}

Tumor angiogenesis is the foundation of tumor growth and metastasis, and in average each tumor blood vessel feeds thousands of tumor cells to maintain their rapid reproduction [1]. Compared with the normal vasculature, the tumor blood vessels usually have abnormal morphology [2], such as incomplete vessel walls, loose cell-cell connections between the tumor endothelial cells, and structurally abnormal basement membrane, etc. [3-7]. Moreover, the blood flow of tumor vasculature is often sluggish and some blood vessels are not continuously perfused [8,9], leading to the observed hyper-permeability and high interstitial fluid pressure in solid tumors [1013]. These unique vascular features of tumor blood vessels naturally provide us a chance to selectively destruct them by developing advanced tumor vascular targeting therapeutic techniques. Because these techniques do not need to directly destruct billions of cancer cells, they are obviously much more effective than the traditional methods for tumor therapy [14]. Aiming at the relatively weaker vascular structure of tumors comparative to that of normal organs, scientists invented some vascular disrupting agents (VDAs) that can disrupt the tumor vasculature through depolymerizing the endothelial microtubules or augmenting local cytokine productions [15-20]. It was revealed that most of the VDAs, such as CA4P, FAA/DMXAA, OXi4503, AVE8062 and ZD6126, indeed efficiently damage the tumor vasculature, but they also show serious cardiotoxicity which largely limits their clinical applications [21].

Recently, we reported a new technique based on the radiofrequency $(\mathrm{RF})$-assisted gadofullerene nanocrystals

\footnotetext{
${ }^{1}$ Beijing National Laboratory for Molecular Sciences, Key Laboratory of Molecular Nanostructure and Nanotechnology, CAS Research / Education Center for Excellence in Molecular Sciences, Institute of Chemistry, Chinese Academy of Sciences, Beijing 100190, China

${ }^{2}$ University of Chinese Academy of Sciences, Beijing 100049, China

${ }^{3}$ CAS Key Lab for Biomedical Effects of Nanomaterials and Nanosafety, National Center for Nanoscience and Technology, Beijing 100190, China

$\dagger$ These authors contributed equally to this study.

* Corresponding authors (emails: zhenmm@iccas.ac.cn (Zhen M); crwang@iccas.ac.cn (Wang C))
} 
(GFNCs) which exhibited high-efficient antitumor activity and low toxicity to normal tissues, and may overcome the big problems of VDAs [22]. Briefly, the gadofullerene based nanomaterials were modified with hydroxyl groups on the surface of carbon cage to form the water soluble GFNCs $[23,24]$. When performing the tumor therapy, the GFNCs solution was intravenously injected to the testing tumor-bearing mice, and partial of the GFNCs particles would reach the tumor blood vessels along with the blood flow and try to penetrate out through the vascular leaks. With the radiation by RF, the tumor vasculature was speculated to be destroyed with the explosive volume expansion of GFNCs in anti-tumor activity but not the magnetocaloric effect [22]. On the contrast, for the vasculature of normal organs, there are no leaks on the blood vessel walls to trap the GFNCs particles, so the phase transition of GFNCs would be harmless. In previous papers, we mainly studied the size expansion mechanism and showed the high efficiency of this technique in tumor therapy. This novel technique showed potential therapeutic advantages for clinical applications, which need to be further investigated.

To unravel the mechanism involved during the tumor vascular disruption, we further made a systematic and detailed investigation on the vascular therapeutic process in this work. Various characterization techniques [25-29] were adopted to monitor the real-time changes of relevant tumor vasculatures and the tumor necrosis by the RF-assisted GFNCs treatment. For example, the fluorescent microscopy technique combined with a mice tumor vascular dorsal skin flap chamber (DSFC) model [30] was employed to observe the vascular morphological changes during the treatment; the magnetic resonance imaging (MRI) was used to evaluate the blood perfusion and vascular permeability of tumor vasculature [31,32]; the dynamic contrast enhanced (DCE) MRI was applied to noninvasively and quantitatively evaluate the vascular function of lesions and the extent of tumor angiogenesis in vivo, which provided valuable physiopathologic information for tumor vascular-disrupting treatments $[33,34]$. The results showed that the RF-assisted GFNCs disrupted the tumor vasculature and induced an irreversible collapse of the tumor vascular net, and thereby the blood supply of tumor vessels was sharply cut off, resulting in extensive tumor necrosis.

\section{EXPERIMENTAL SECTION}

\section{Materials}

All reagents and solvents were obtained commercially and used without further purification. Solid Gd@ $\mathrm{C}_{82}(99 \%$ purity) was purchased from Xiamen Funano Co. Ltd. (Xiamen, China). The $30 \%$ hydrogen peroxide $\left(\mathrm{H}_{2} \mathrm{O}_{2}\right.$, A.R.) and ethanol $\left(\mathrm{C}_{2} \mathrm{H}_{5} \mathrm{OH}, \mathrm{A} . \mathrm{R}\right.$.) were purchased from Beijing Chemical Works, China. Fluorescein isothiocyanate (FITC)-dextran (average molecular weight40,000) and Rhodamine B isothiocyanate-dextran (average molecular weight-70,000) were purchased from Sigma-Aldrich (St. Louis, MO, USA). Gadopentetate dimeglumine injection (Gd-DTPA) $(15 \mathrm{~mL}, 7.04 \mathrm{~g})$ was purchased from Bayer Schering Pharma AG, Germany. Pentobarbital sodium salt was purchased from SigmaAldrich. Isoflurane was purchased from RWD Life Science Co. Ltd. (Shenzhen, China). The DSFC device was purchased from APJ Trading Co. Inc., USA.

\section{Preparation and characterization of the GFNCs}

The water-soluble GFNCs were synthesized via a facile solid-liquid reaction as previously reported [35]. The solid Gd@ $\mathrm{C}_{82}$ was directly added to the hydroxyl moieties $\mathrm{H}_{2} \mathrm{O}_{2}$ by nucleophilic additions in alkaline conditions. The mixture was vigorously stirred for $40 \mathrm{~min}$ at $50^{\circ} \mathrm{C}$. The suspension obtained was washed with ethanol three times and then dialyzed overnight in ultrapure water.

The purity of the as-prepared GFNCs $(20 \mu \mathrm{L}$, $1.0 \mathrm{mg} \mathrm{mL}^{-1}$ ) was verified on the Cosmosil column by high performance liquid chromatography (HPLC, LC10A, Shimadzu, Japan) with an evaporative lightscattering detector $\left(80^{\circ} \mathrm{C}, 3.5\right.$ bar of nitrogen pressure, sedex 75$)$. The mobile phase was $90 \%$ acetonitrile in water with a flow rate of $1.0 \mathrm{~mL} \mathrm{~min}{ }^{-1}$. The GFNCs were further characterized using Fourier transform infrared spectroscopy (FTIR, Nicolet iN10TM, Thermo Scientific, USA). The size of the GFNCs in pure water was measured with atomic force microscopy (AFM, Nano Wizard 4 Nano Science, JPK, Germany) and dynamic light scattering (DLS, ZS90, Malvern, UK).

\section{The $300 \mathrm{MHz}$ radiofrequency generator}

The RF generator used was the standard equipped $300 \mathrm{MHz}$ resonance frequency generator in a 7T MRI scanner (BioSpec70/20 USR, Bruker, Germany). The treatment was applied with a $2 \mathrm{D} \mathrm{T} 1$ weighted sequence: repetition time $(\mathrm{TR})=300 \mathrm{~ms}$, echo time $(\mathrm{TE})=6.06 \mathrm{~ms}$, number of excitations $(\mathrm{NEX})=10$, field of view $(\mathrm{FOV})=$ $35 \times 35 \mathrm{~mm}$, flip angle $(\mathrm{FA})=90$, matrix $(\mathrm{MTX})=$ $256 \times 256$ and $1 \mathrm{~mm}$ slice thickness. The resolution was $0.137 \times 0.137 \mathrm{~mm}$. The tumor-bearing mice were placed in the center of the main magnet and volume radio frequency coil. 
To conveniently compare experiments in the DSFC model, a home-made $300 \mathrm{MHz}$ radiofrequency generator was included in the commercial MXG Analog Signal Generator (100 kHz ${ }^{-1} \mathrm{GHz}, \mathrm{N} 5181 \mathrm{~A}$, Agilent, USA), and a signal amplifier BLAXH 20 (Bruker, Germany) and a self-designed small RF coil were used on the DSFC device. The RF power ( $1 \mathrm{dBm}, 10 \%$ duty circle) could easily be monitored by the spectrum analyzer (DSA 815, RIGOL, China).

\section{Animal models}

All animal studies were undertaken in accordance with national and local ethical committee regulations. This study was performed on BALB/c mice for the DCE-MRI and nude mice for the DSFC model (female, 4-5 weeks, weighing 18-20 g), which were both housed in a standard pathogen-free room at the Institute of Process Engineering, Chinese Academy of Sciences. Hepatoma 22 cells (H22) $\left(5 \times 10^{6}\right.$ tumor cells in $100 \mu \mathrm{L}$ of saline) were injected subcutaneously into the mice in the right rear flank area. The experiments were conducted when the tumors reached a volume of $100 \mathrm{~mm}^{3} 5-7$ days after the tumor inoculation. The tumors were irradiated in the $300 \mathrm{MHz}$ RF generator for $1.5 \mathrm{~h}$ via an intravenous injection of GFNC solution ( $50 \mathrm{mg} \mathrm{kg}^{-1}$ ) for treatment and using saline as a control.

\section{DSFC model}

For the DSFC model, the window chambers were surgically implanted into $\mathrm{BALB} / \mathrm{c}$ nude mice as previously described with slight modification [36]. The experiments were performed under aseptic conditions in a specific pathogen-free environment.

During the operation, the mice were anesthetized with $2 \%$ pentobarbital sodium $(100 \mu \mathrm{L})$. The entire back of the mouse was disinfected with iodophor, and two symmetrical titanium frames (with diameters of $27 \mathrm{~mm}$ ) were implanted to sandwich the skin. The skin and the chambers were sutured together with 6.0 nylon around the windows so that as many blood vessels as possible could be located in the center of the window and fixed without influence from the beating of the heart or breathing. One layer of the skin was removed from a circular area approximately $15 \mathrm{~mm}$ in diameter, and the remaining layer was covered with a microscope glass slip in diameter of $12 \mathrm{~mm}$, which was incorporated into one of the frames. The exposed skin was then placed surface down in sterile neutral saline. During the surgery, suspended Hepatoma 22 cells (H22) $\left(5 \times 10^{7} \mathrm{~mL}^{-1}\right)$ were prepared and $10 \mu \mathrm{L}$ was inoculated in the skin muscle closed to the vessels in the DSFC. Then, the mice with DSFC tumors were given the antibiotic amoxicillin in their drinking water ( $250 \mathrm{mg}$ per $5 \mathrm{~mL}$ of water) and were maintained at $23^{\circ} \mathrm{C}$ and $50 \%$ humidity with free access to food and water and standard $12 \mathrm{~h}$ light/dark cycles.

All the images of tumor vasculature and normal vasculature were acquired at 5-7 days after $\mathrm{H} 22$ tumor inoculation and DSFC transplantation. The mice bearing DSFC were anaesthetized via isoflurane inhaled through a nose cone and immobilized onto the microscope stage using a custom platform to minimize motion from breathing and circulation for real-time imaging.

Real-time optical imaging of blood vessels in DSFC model Blood vessels in DSFC models were imaged directly and stably on a custom platform for bright field imaging by the upright optical microscope (OPSTEC, CCD: SONY ICX452AQ) at $\times 4$ objective.

For assessing the morphological change of tumor vasculature under RF-assisted GFNCs treatment, the control cohort of only RF treated ones (RF-control) in tumor vessels was set and the cohort of RF-assisted GFNCs treated ones in normal tissue vessels was also set for morphological change research.

\section{Real-time fluorescence imaging of blood vessels in DSFC model}

The intravital fluorescence imaging of vasculature was performed using an upright fluorescence microscope (Nikon ECLIPSE Ni-U, Japan) with a $20 \times$ objective. Fluorescein isothiocyanate-dextran $(40 \mathrm{kDa}, 75 \mu \mathrm{L}$ of $25 \mathrm{mg} \mathrm{mL}^{-1}$ ) was intravenously injected into the mice to label the blood vessels. The excitation filter of $475 \pm$ $25 \mathrm{~nm}$ on the fluorescent tube turntable (NI-FLT6) switch was used for FITC channel.

For assessing the tumor vascular change under RF assisted GFNCs treatment (Treatment), only RF treated group (RF-control) in tumor blood vessels were as control. In addition, the group of RF assisted GFNCs treated normal tissue vasculature was used as other control group for studying the effect of RF-assisted GFNCs treatment on normal vasculature.

\section{Fluorescence imaging of tumor sections}

We visualized tumor blood vessels in the treatment group using 2D-fluorescence imaging performed with an upright fluorescence microscope (Nikon ECLIPSE Ni-U, Japan). Rhodamine B isothiocyanate-dextran (70 kDa, $150 \mu \mathrm{L}$ of $5 \mathrm{mg} \mathrm{mL}^{-1}$ ) was intravenously injected into the mice to label the blood vessels. The tumor was carefully 
dissected within 10-15 min after Rhodamine B isothiocyanate-dextran injection, immediately immersed in optimum cutting temperature (OCT) compound and frozen at $-20^{\circ} \mathrm{C}$ for $10 \mathrm{~min}$. A series of $50 \mu \mathrm{m}$ frozen sections from central regions of each tumor were sliced using microtome cryostat (Leica CM1950, Germany) and imaged immediately with a $20 \times$ objective. Excitation of rhodamine $\mathrm{B}$ was performed with the filter of $575 \pm$ $25 \mathrm{~nm}$ on the fluorescent tube turntable (NI-FLT6) switch.

\section{DCE-MRI}

The DCE-MRI experiments were performed on a 7T MRI system (BioSpec 70/20 USR, Bruker, Germany). The mice were anaesthetized with a $2 \%$ isoflurane-oxygen mixture in an isoflurane induction chamber, as this form of anesthesia provides concentration-dependent rapid general anesthesia and quick recovery after the discontinuation of isoflurane administration [37]. Body temperature was maintained at $37^{\circ} \mathrm{C}$ with a circulating water blanket and was monitored together with the mice breathing during the experiment.

The $\mathrm{T} 1$ weighted gradient echo images were obtained with a dynamic contrast enhancement fast low angle shot (DCE-FLASH) sequence using the following parameters: $\mathrm{TR} / \mathrm{TE}=96.67 / 3 \mathrm{~ms}, \mathrm{FA}=15, \mathrm{FOV}=35 \times 35 \mathrm{~mm}$, $\mathrm{MTX}=128 \times 128$, temporal resolution $=10.247 \mathrm{~s}$ and $\mathrm{cycle}$ $=100$. After the fifth scan, the commercial MRI contrast agent Gd-DTPA was intravenously administered over $2 \mathrm{~s}$

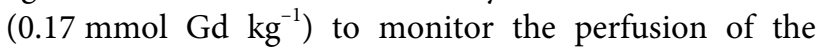
contrast agent in 95 continued images. To calculate the proton density values, five T1-FLASH images were acquired before DCE-MRI sequences using the following parameters respectively: $\mathrm{TR} / \mathrm{TE}=150 / 2.074 \mathrm{~ms} ; \mathrm{FA}=10$, 15, 20, 25 and 30; FOV=35×35 mm; and MTX $=128 \times 128$.

Six BALB/c mice were divided randomly into two groups: the Treatment group and the RF-control group (intravenous injection with saline and irradiation with $\mathrm{RF})$. The experiments were conducted when the tumors reached a volume of $100 \mathrm{~mm}^{3} 5-7$ days after tumor inoculation.

\section{DCE-MRI data analysis}

We reconstructed and analyzed the FLASH images with the MRICRON software. The dynamic FLASH data in DICOM format were processed using home-made programs in MATLAB. Regions of interest (ROIs) were manually drawn at the periphery of the middle tumor slice in each mouse for kinetic analysis because the tumor periphery is highly vascularized with angiogenic capil- laries [38]. For the blood signal intensity $(S)$ or arterial input function, ROIs were limited to the artery sections in normal tissue beside the tumor. The average signal intensity of the pre-contrast images $\left(S_{0}\right)$ in the ROIs was used as the pretreatment value. The signal enhancement after contrast injection was calculated as follows:

$$
\Delta S_{t}=\frac{S_{t}-S_{0}}{S_{0}}
$$

where $S_{t}$ is the signal intensity at time $t$. The DCE-MRI data were converted into the concentration of the contrast agent using the different flip angle method [39]. The general equation for $S$ values is as follows:

$$
S(\alpha)=\frac{M_{0}\left(1-e^{-\frac{-\mathrm{TR}}{-\mathrm{T} 1}}\right) \sin \alpha}{\left(1-\cos \alpha e^{-\frac{-\mathrm{TR}}{-\mathrm{T} 1}}\right)},
$$

where $\alpha$ is the flip angle, TR is the repetition time, and $M_{0}$ is the equilibrium longitudinal magnetization. The T1 and $M_{0}$ parameters were estimated with curve fitting with the nonlinear Levenberg-Marquardt Least Squares method using MR images at different flip angles $\left(10^{\circ}, 15^{\circ}\right.$, $20^{\circ}, 25^{\circ}$ and $30^{\circ}$ ). Following the estimation of $M_{0}$ and T1 (0) values for the pre-contrast image, the post-contrast T1 value $(\mathrm{T} 1(t))$ can be estimated as a function of time from the $S(t)$ for the post-contrast image with $\alpha=15^{\circ}$.

The concentration of the contrast agent was calculated by the determination of the difference in the longitudinal relaxation rate, as follows:

$$
\frac{1}{\mathrm{~T} 1(t)}=\frac{1}{\mathrm{~T} 1(0)}+C(t) \cdot r 1 \text {. }
$$

The measured tissue concentration time course was individually fitted to the Tofts-Kety Model:

$$
C_{\mathrm{t}}(t)=v_{\mathrm{p}} C_{\mathrm{p}}(t)+K_{\text {trans }} \int C_{\mathrm{p}}(\tau) \mathrm{d} \tau,
$$

where $K_{\text {trans }}$ is the volume transfer constant between the blood plasma and extravascular extracellular space (EES) $\left(\min ^{-1}\right), v_{\mathrm{p}}$ is the blood plasma volume per volume unit of tissue, $C_{t}$ is the tracer concentration in the tumor tissue and $C_{\mathrm{p}}$ is the tracer concentration in the arterial blood plasma.

\section{RESULTS AND DISCUSSION}

\section{Synthesis and characterizations of GFNCs}

GFNCs were synthesized through a solid-liquid reaction by directly introducing the hydroxyl moieties onto solid $\mathrm{Gd} @ \mathrm{C}_{82}$ with nucleophilic additions of $\mathrm{H}_{2} \mathrm{O}_{2}$. GFNCs were dialyzed against ultrapure water for $72 \mathrm{~h}$ and then verified by HPLC using a Cosmosil column, and the re- 

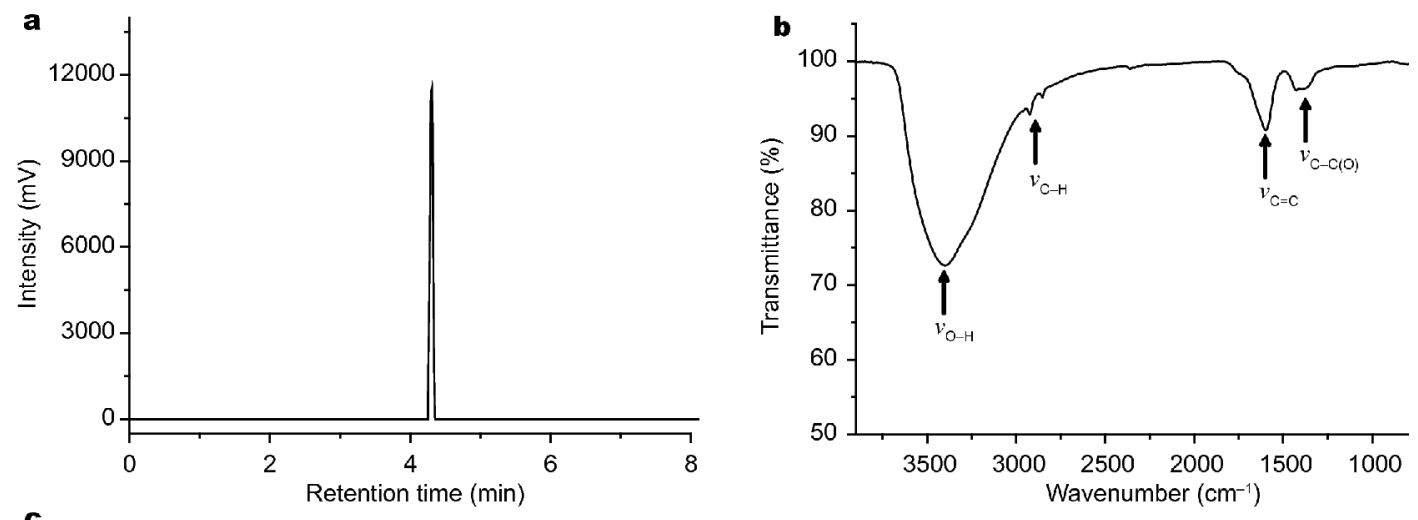

C
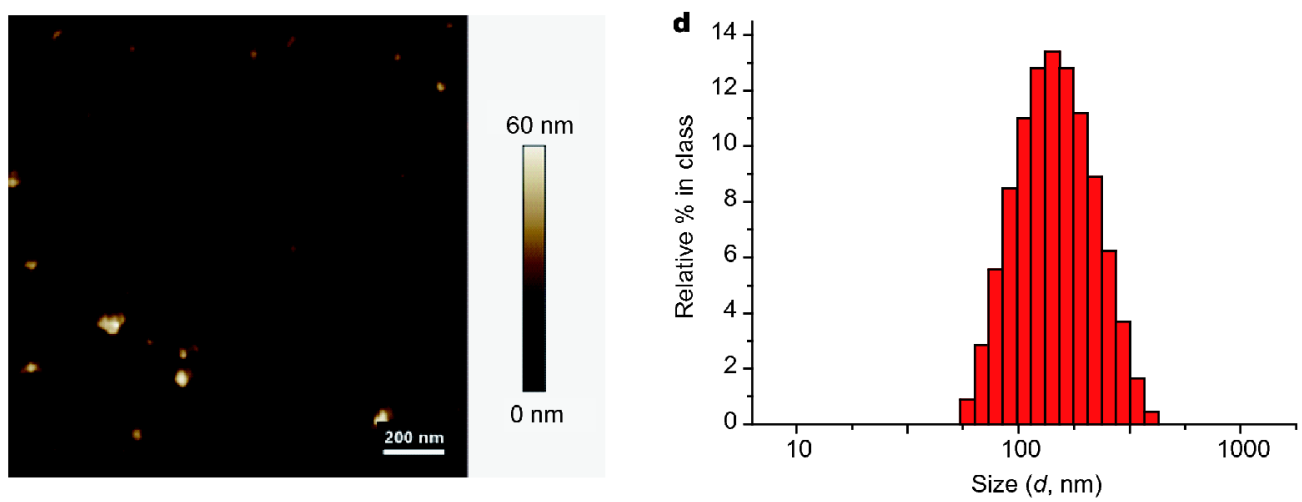

Figure 1 The physiochemical characterizations of GFNCs. (a) Chromatography of the purified GFNCs on a Cosmosil column (4.6×250 mm, flow rate $1.0 \mathrm{mg} \mathrm{mL}^{-1}, 90 \%$ acetonitrile in water as the eluent). The retention time of GFNCs was 4.3 min achieving a good symmetrical peak shape. (b) The FTIR spectrum of GFNCs. (c) AFM image of GFNCs aggregates. The average height and diameter were approximately 30 and 80 nm, respectively. (d) The measured hydrodynamic size distribution of GFNCs in ultrapure water by DLS. The size was $157.2 \mathrm{~nm}$.

tention time was $4.3 \mathrm{~min}$ (Fig. 1a). A peak with good symmetry appeared in the chromatograph, suggesting that the unreacted reagents were already removed and the as-prepared GFNCs were fully purified. As shown in Fig. $1 b$, the FTIR spectrum of GFNCs exhibits several peaks, in which the main peak at $3,400 \mathrm{~cm}^{-1}$ is assigned to the characteristic vibration bands of $-\mathrm{OH}$ and the middle peak at $1,598 \mathrm{~cm}^{-1}$ belongs to the skeletal vibration frequency of $\mathrm{C}=\mathrm{C}$ of fullerenes. The peaks locating at $1,465-1,340 \mathrm{~cm}^{-1}$ are attributed to the asymmetric stretching bands of $-\mathrm{C}-\mathrm{OM}(\mathrm{M}=\mathrm{H}, \mathrm{C})$, suggesting the existence of $-\mathrm{C}-\mathrm{OH}$ and $-\mathrm{C}-\mathrm{O}-\mathrm{C}$ groups $[40,41]$. The height and the diameter of GFNCs were approximately $30 \mathrm{~nm}$ and $80 \mathrm{~nm}$ as observed by AFM (Fig. 1c and Fig. $\mathrm{S} 1$ ). The hydrodynamic size (approximately $157.2 \mathrm{~nm}$ ) of GFNCs in aqueous solution measured by the dynamic light scattering (DLS) was obviously enlarged due to the coverage of the solvent layer (Fig. 1d). GFNCs exhibited uniform distribution with a polydispersity index (PDI) of 0.202 and promoted stability with a highly negative zeta potential of $-39.5 \mathrm{mV}$ in ultrapure water.

\section{Morphological and functional observation of the tumor} vascular disruption by RF-assisted GFNCs treatment The detail of the morphological and functional observation in tumor vasculature by RF-assisted GFNCs treatment was taken in a H22 tumor bearing DSFC model and combined with a RF coil. As shown in Fig. 2a, neovascularization was obviously visualized at $7^{\text {th }}$ day after tumor inoculation. A home-made $300 \mathrm{MHz}$ RF coil was equipped on the back of DSFC for RF irradiation (Fig. 2b).

First, the short-time observation of the tumor blood vessels was performed using vascular fluorescent perfusion imaging in the $\mathrm{H} 22$ tumor bearing DSFC model. The blood vessels of normal tissue in chamber treated by RFassisted GFNCs were studied as a control. FITC-dextran (70 kDa; $75 \mu \mathrm{L}$ of $25 \mathrm{mg} \mathrm{mL}^{-1}$ ), a commonly used blood vessel marker, was intravenously pre-injected to label the vasculature. In the RF-assisted GFNCs treatment group, the tumor blood vessels were observed to gradually slow down with the reduced fluorescence perfusion within 15 min of treatment (Fig. 2c). Instantly, the blood cells 
a

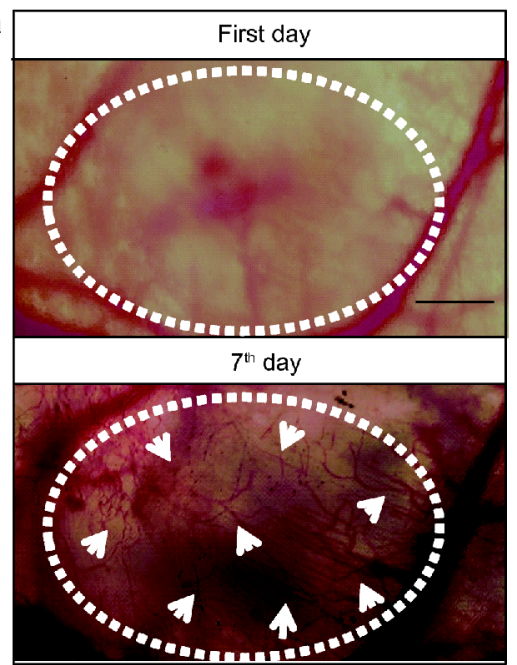

b

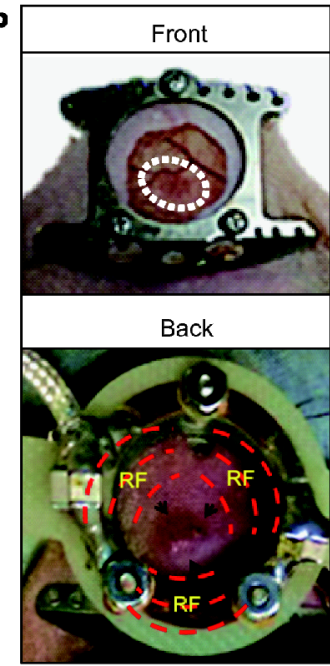

C
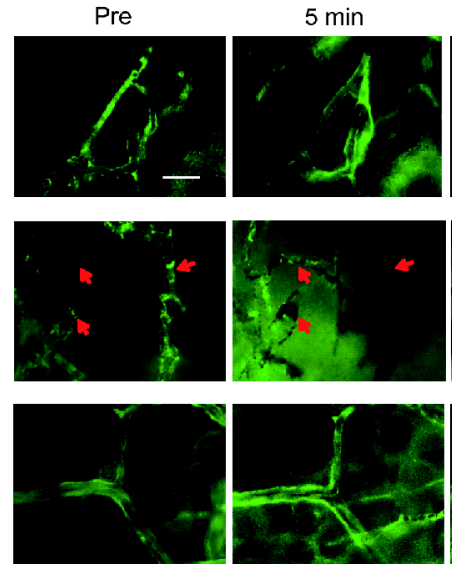

$5 \mathrm{~min}$
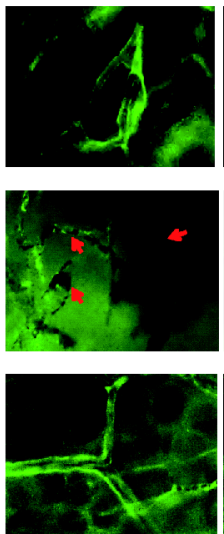

$15 \mathrm{~min}$
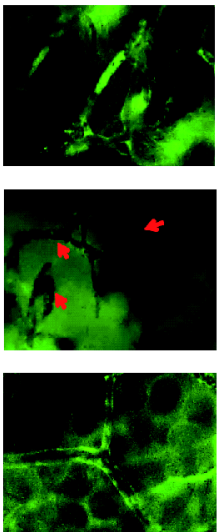
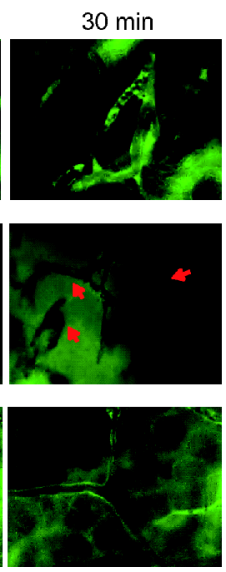

$60 \mathrm{~min}$
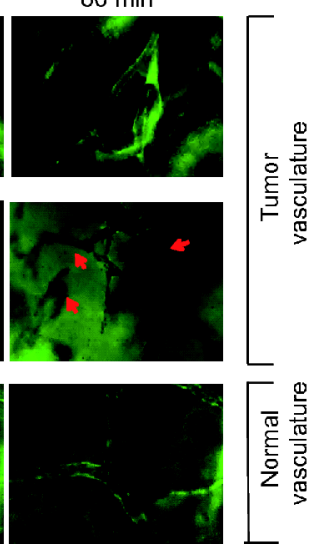

Figure 2 Short-time observation of the tumor vasculature during RF-assisted GFNCs treatment in DSFC model in vivo. (a) The first day and the $7^{\text {th }}$ day after $\mathrm{H} 22$ tumor inoculation in DSFC. The white dot line and white arrows indicated the approximate border of tumor and tumor angiogenesis, respectively. The scale bar is $300 \mu \mathrm{m}$. (b) The RF coil is combined with the DSFC model in the back to irradiate the corresponding RF to trigger the GFNCs treatment. The black arrows indicated the solid tumor tissues in back of DSFC. (c) The fluorescence images of vasculature in RF-control, treatment in tumor and normal tissue $(n=3)$. In the treatment group, the most majority of tumor vasculature was out of circulations at 15 min of treatment compared to the respective controls. The scale bar is $50 \mu \mathrm{m}$.

gradually aggregated and then the flow of the whole blood vessels sequentially shut off without perfusion of the FITC-dextran in treatment group from 15 to 60 min (Fig. $2 c$, highlighted by red arrows). There was little influence on either tumor blood vessels in the RF alone group or normal blood vessels treated by RF-assisted GFNCs. In this study, it was significant that the RF-assisted GFNCs treatment leads to rapid blood flow shut off in tumor during treatment.

The typical destruction process of $\mathrm{H} 22$ tumor vascular morphology treated by RF-assisted GFNCs within $48 \mathrm{~h}$ was shown in Fig. 3a. The tumor vasculature in some areas (such as ROI. I, ROI. II and ROI. III highlighted by white dotted circles in Fig. 3b) began to be broken and hemorrhaged during the treatment. With the time extending, the areas of hemorrhage of the tumor vasculature were continually increased in the three ROIs after treatment, leading to the remarkable collapse and fragmentation of the whole tumor vascular net. Within $24 \mathrm{~h}$ after treatment, the hemorrhagic damage and the decrease of the tumor blood vessels were continued. Vascular hemorrhage stopped and no recovery was observed at $48 \mathrm{~h}$. Thus, the $\mathrm{H} 22$ tumor finally become necrotic by the rapid and persistent vascular collapse in morphology after the RF-assisted GFNCs treatment.

In addition, the structure of tumor vascular network could not be disrupted by RF treatment without administration of GFNCs. The tumor capillary blood vessels in 


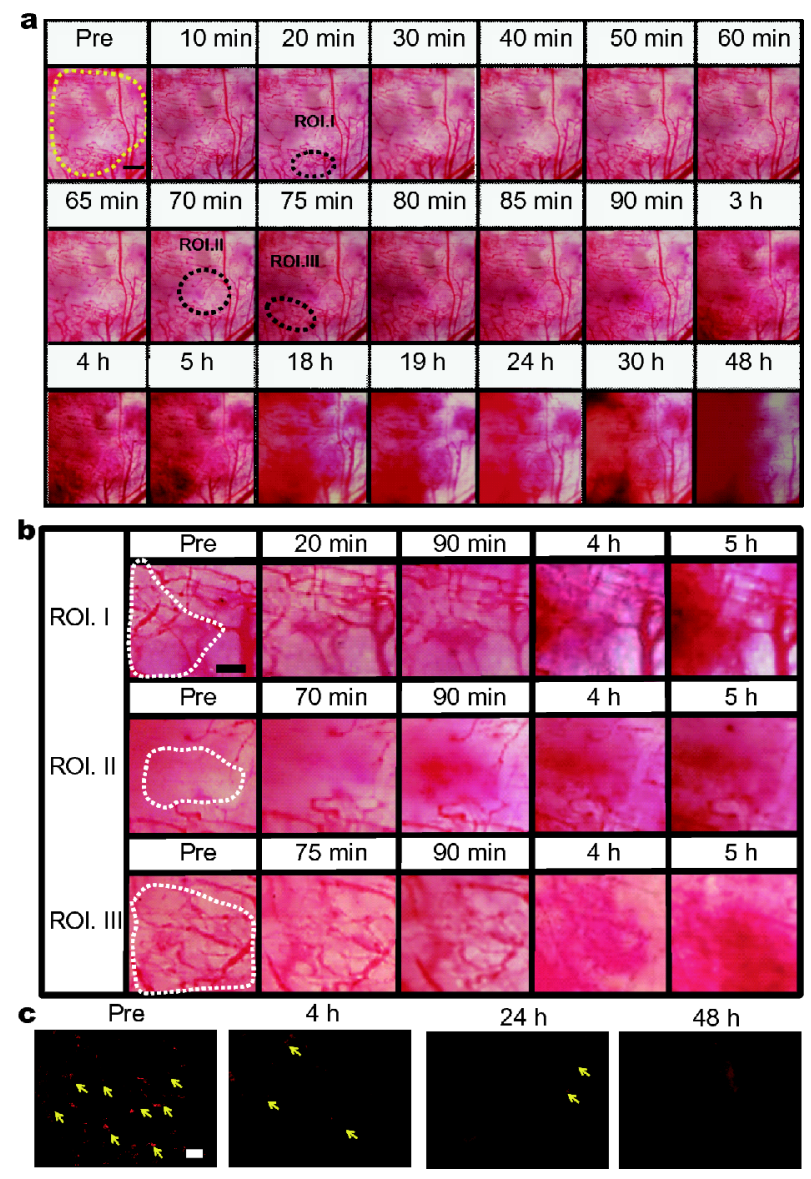

Figure 3 Morphological and functional observation of the tumor vascular disruption by RF-assisted GFNCs treatment. (a) Real-time observations of the typical tumor vascular destruction by RF-assistant GFNCs treatment at separate time points obtained at $40 \times$ magnification in the DSFC model. The yellow dotted lines and the black ones indicate the approximate border of tumor and ROI of tumor vasculature, respectively. The scale bar is $300 \mu \mathrm{m}$. (b) The amplification of three representative ROIs of tumor vasculatures in DSFC. The white dotted circles indicate the tumor vasculature impacted by RF-assistant GFNCs treatment. The scale bar is $100 \mu \mathrm{m}$. (c) Fluorescence imaging of rhodamine $B$ isothiocyanate-dextran $\left(70 \mathrm{kDa}, 150 \mu \mathrm{L}, 5 \mathrm{mg} \mathrm{mL}^{-1}\right)$ by intravenous injection shows decrease of blood vessels in tumor $(n=3)$ within $48 \mathrm{~h}$ after treatment. Yellow arrows indicate the tumor vasculature perfused by rhodamine B dextran. Scale bar is $50 \mu \mathrm{m}$.

the RF-control group (Fig. S2) maintained good integrity under irradiation of $300 \mathrm{MHz} \mathrm{RF}$ for $1.5 \mathrm{~h}$ and minor change was found within $48 \mathrm{~h}$ after treatment. Moreover, it is obvious that the structure of normal vascular system was not disrupted under the RF assisted GFNCs treatment, which avoided damage to normal vessels to a large degree (Fig. S3). Accordingly, the tissue pathological analysis in the previous report exhibited that the RF assisted GFNCs displayed negligible damage to the normal tissues, even to the liver tissue with higher accumulation of GFNCs [22].

To further evaluate the long-term potential of RF assisted GFNCs to induce functional damage of the whole vascular net in $\mathrm{H} 22$ tumor xenografts, 2D-fluorescence perfusion imaging of the tumor vasculature was performed at the $4^{\text {th }}, 24^{\text {th }}$ and $48^{\text {th }}$ h post treatment. A standard macromolecular vessel marker, Rhodamine B isothiocyanate-dextran, was pre-injected intravenously into the mice to label the tumor vessels by perfusion and the frozen tumor sections were dissected from the mice for imaging. As shown in Fig. 3c, large degrees of reduction of perfused vessels were clearly observed due to the disruption of tumor vasculature in the treatment group. Compared with pre-treatment, the number of tumor blood vessels perfused was sequentially decreased at the $4^{\text {th }}$ and $24^{\text {th }} \mathrm{h}$ with little change at the $48^{\text {th }} \mathrm{h}$ posttreatment (Fig. 3c). A remarkable drop in vascular perfusion was followed after treatment, indicating an obvious and permanent damage in function of the whole tumor vascular net.

In this study, it was significant that RF-assisted GFNCs could cause the rapid and specific hemorrhage and collapse of the partial tumor blood vessels due to the breakdown and abscission from the basilemma of endothelia cell in tumor vasculature (Fig. S4) [22], efficiently leading to functional impairment of the whole tumor vascular net.

\section{Quantification of disruption in tumor vascular function by RF-assisted GFNCs treatment using DCE-MRI in vivo} The DCE-MRI is a "gold standard" method to evaluate functional characteristics of the vascular net, giving out the information in the blood perfusion and the vascular permeability $[42,43]$. Thus, we performed the quantitative DCE-MRI to assess tumor microvascular function after RF-assisted GFNCs treatment (Fig. 4a and b). The H22 tumors (yellow dotted circles) were injected with GdDTPA and imaged with the DCE-FLASH sequence at different time points of treatment (Fig. $4 \mathrm{c}$ and d). Before the treatment, a rapid and great increase of the MRI signals after the Gd-DTPA injection was observed both in the RF-control group and RF-assisted GFNCs treatment group. While, in RF-assisted GFNCs treatment group, the increase of MRI signal intensity in the tumors was visibly lower compared with the RF-control group after GdDTPA injection (Fig. 4d). Meanwhile, the concentration of the Gd-DTPA in the periphery of the H22 tumors before and after treatment at different time points was calculated for the RF-control and RF-assisted GFNCs 

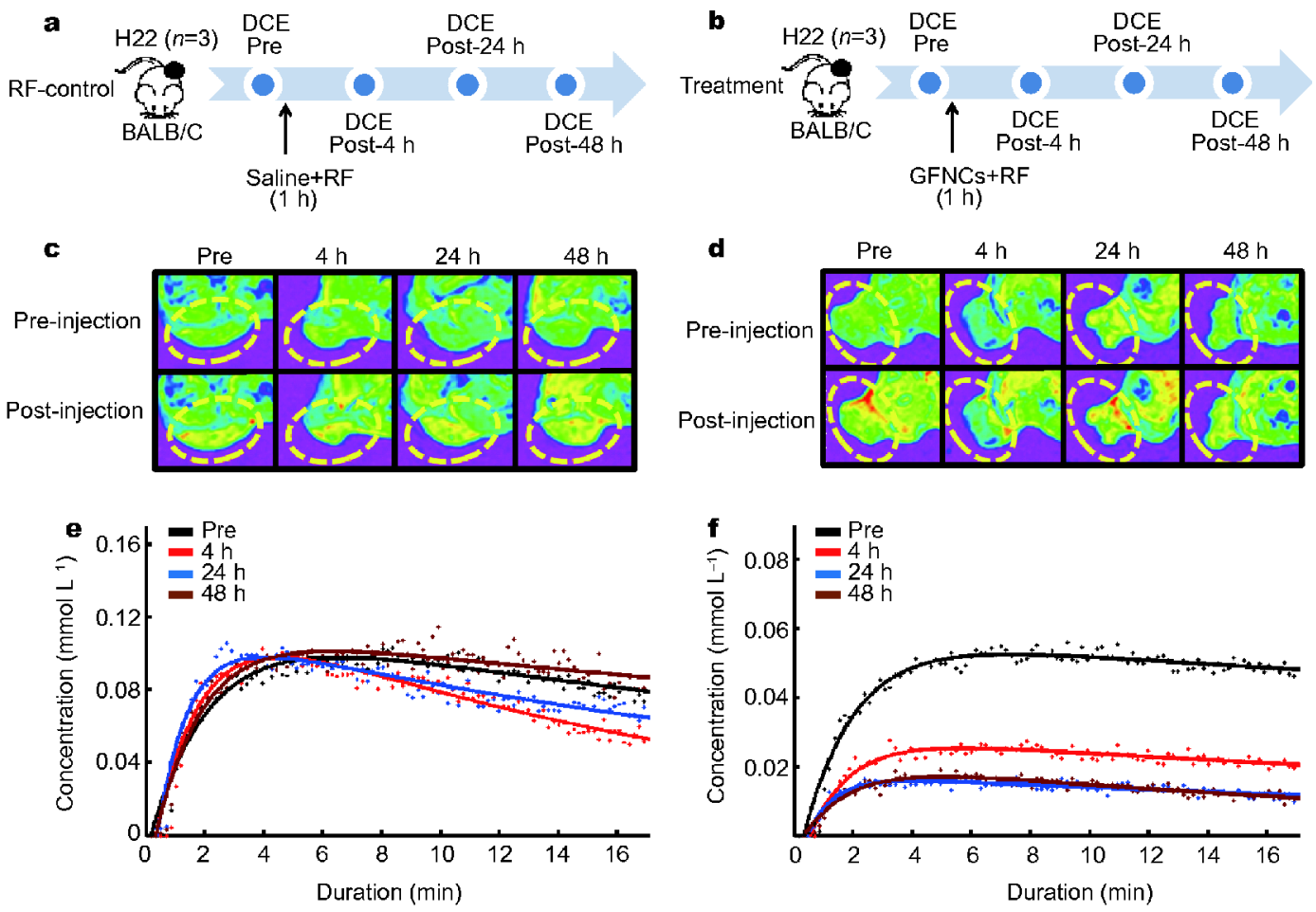

Figure 4 The signal and concentration of Gd-DTPA in duration of DCE-MRI in periphery of H22 tumor. (a, b) The procedure of DCE-MRI experiment of RF-Control (a) and Treatment (b) $(n=3)$. (c, d) DCE-MRI in the duration of Gd-DTPA injection at different treatment time points in RF-control (c) and Treatment (d). The enhancement of MRI signal in tumor before and after contrast agent injection in treatment was obviously smaller than that in RF-control. The yellow dotted circles indicate the location of tumor. (e, f) The concentration of Gd-DTPA in periphery of H22 tumor in duration of DCE-MRI in RF-control (e) and Treatment (f) at different treatment times. The DCE-MRI data were converted into the concentration of the contrast agent using a different flip angle method.

treatment groups, and the representative concentrationtime curves were fitted in Fig. $4 \mathrm{e}$ and $\mathrm{f}$. There was little change in the concentration of Gd-DTPA at different time points for the RF-control group. In the treatment group, slower and lower increase of the concentration of Gd-DTPA was observed with the decreased perfusion and no recovery until the $48^{\text {th }} \mathrm{h}$, indicating the functional damage of tumor blood vessels after RF-assisted GFNCs treatment.

After that, a pharmacokinetic parameter, $K_{\text {trans }}$ (the rate of transfer of the contrast agent from the blood to the interstitial space), was calculated from the DCE-MRI data using the classical Tofts-Kety model with homemade programs written in Matrix Laboratory (MATLAB). The value of $K_{\text {trans }}$ depends on the balance between capillary permeability and the blood flow in the tumor [44]. The $\Delta K_{\text {trans }}$ values of the tumor boundary capillary at different treatment time points in two groups were shown in Fig. 5a. After RF-assisted GFNCs treatment, the values of $K_{\text {trans }}$ were dramatically decreased by $66.68 \%, 78.64 \%$ and $78.93 \%$ at the $4^{\text {th }}, 24^{\text {th }}$ and $48^{\text {th }}$ h post-treatment, respec- tively. In this study, the permeation of Gd-DTPA was significantly reduced and the concentration of Gd-DTPA displayed smaller and slower increments after treatment. As a result, the $K_{\text {trans }}$ values of $\mathrm{H} 22$ tumors were greatly and persistently reduced within $24 \mathrm{~h}$ after RF-assisted GFNC treatment and little change with no recovery at $48 \mathrm{~h}$, suggesting considerable and permanent damage to the capillary function of the tumor.

However, as for the RF-control group, the DCE-MRI parameters $K_{\text {trans }}$ of the whole tumor exhibited slight increase at the $4^{\text {th }}, 24^{\text {th }}$ and $48^{\text {th }} \mathrm{h}$ post-treatment (increased by approximately $10.86 \%, 17.47 \%$ and $12.27 \%$, respectively) because of neovascularization, suggesting that the RF did not disrupt the function of tumor vessels in mice without the administration of GFNCs. It was more visually displayed that the value of $K_{\text {trans }}$ in the whole tumor was markedly decreased after RF-assisted GFNCs treatment compared to the RF control group in $K_{\text {trans }}$ mapping (Fig. 5b). As a result, the supply of oxygen and nutrients for tumor was eventually cut off in the whole tumor vascular network after RF-assisted GFNCs treatment due 

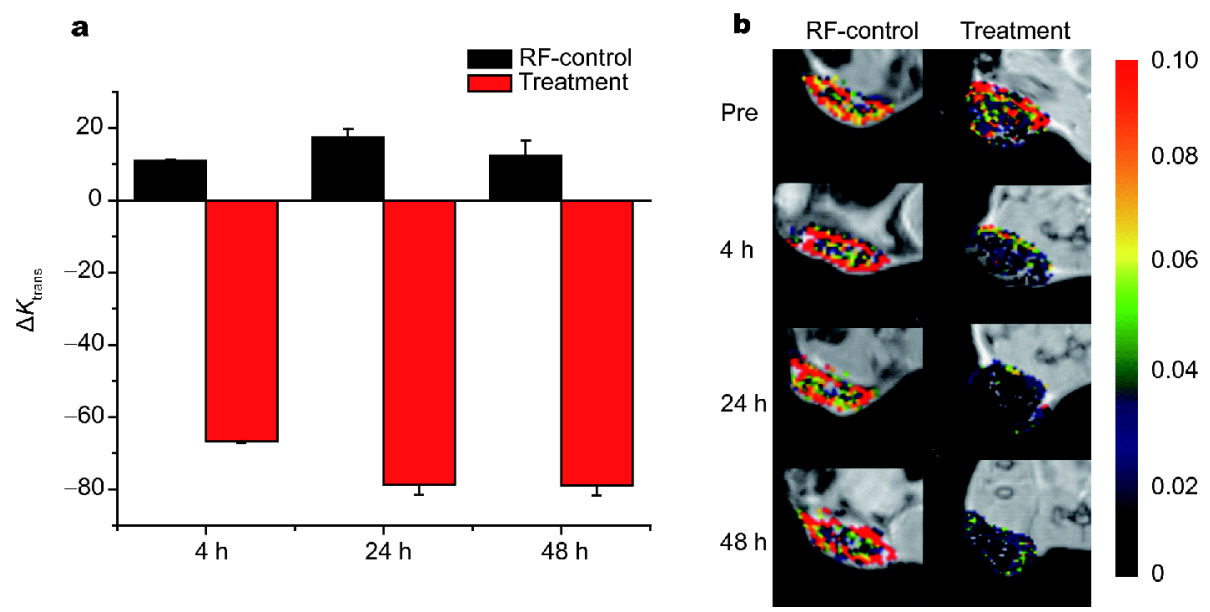

Figure 5 Hemodynamic parameter $K_{\text {trans }}$ of tumor in DCE-MRI at the pre-treatment, $4^{\text {th }}, 24^{\text {th }}$ and $48^{\text {th }} \mathrm{h}$ after treatment. (a) $\Delta K_{\text {trans }}$ (relative change in $K_{\text {trans }}$ between before and after treatment) of the periphery of H22 tumors $(n=3)$ in RF-control and Treatment at the $4^{\text {th }}, 24^{\text {th }}$ and $48^{\text {th }}$ h of posttreatment $\left(\Delta K_{\text {trans }}=\left(K_{\text {trans }}(\mathrm{t})-K_{\text {trans }}(\right.\right.$ pre $\left.)\right) / K_{\text {trans }}($ pre $\left.) \times 100 \%\right)$. These data were expressed as mean \pm SD. (b) $K_{\text {trans }}$ map of H 22 tumor for RF-control and Treatment at the pre-treatment, $4^{\text {th }}, 24^{\text {th }}$ and $48^{\text {th }} \mathrm{h}$ after treatment. Colored parts and gray parts are the manually-defined tumor regions and the normal muscle tissue beside tumor, respectively.

to the outstanding vascular disrupting effects, leading to an accelerated ischemic necrosis of tumor.

\section{CONCLUSIONS}

In summary, we first displayed the real-time process of the tumor vascular disruption by RF-assisted GFNCs treatment qualitatively and quantitatively. The rapid and selective disintegration in morphology of local tumor vasculature had been real-time observed in the DSFC model, followed by a persistent and extensive destruction in function of the whole corresponding tumor vasculature which was quantified with the DCE-MRI. After the RFassisted GFNCs treatment, several tumor blood vessels were shut off and ruptured, leading to the collapse of whole tumor vascular network, which finally caused the tumor ischemic necrosis. These results indicate a clear mechanism of RF-assisted GFNCs treatment based on tumor vascular disruption, which is beneficial for the clinical application of this technique in the future.

Received 23 December 2017; accepted 29 January 2018; published online 11 February 2018

1 Margulis K, Neofytou EA, Beygui RE, et al. Celecoxib nanoparticles for therapeutic angiogenesis. ACS Nano, 2015, 9: 94169426

2 Nagy JA, Dvorak HF. Heterogeneity of the tumor vasculature: the need for new tumor blood vessel type-specific targets. Clin Exp Metastasis, 2012, 29: 657-662

3 Voronin DV, Sindeeva OA, Kurochkin MA, et al. In vitro and in vivo visualization and trapping of fluorescent magnetic microcapsules in a bloodstream. ACS Appl Mater Interfaces, 2017, 9:
6885-6893

4 Liotta LA, Stetler-Stevenson WG. Tumor invasion and metastasis: an imbalance of positive and negative regulation. Cancer Res, 1991, 51: 5054-5059

5 Dvorak HF, Nagy JA, Dvorak J, et al. Identification and characterization of the blood vessels of solid tumors that are leaky to circulating macromolecules. Am J Pathol, 1988, 133: 95-109

6 Kobayashi H, Tsuruchi N, Sugihara K, et al. Expression of $\alpha-$ smooth muscle actin in benign or malignant ovarian tumors. Gynecologic Oncology, 1993, 48: 308-313

7 Tozer GM, Kanthou C, Baguley BC. Disrupting tumour blood vessels. Nat Rev Cancer, 2005, 5: 423-435

8 Tozer G, Lewis S, Michalowski A, et al. The relationship between regional variations in blood flow and histology in a transplanted rat fibrosarcoma. Br J Cancer, 1990, 61: 250-257

9 Carmeliet P, Jain RK. Angiogenesis in cancer and other diseases. Nature, 2000, 407: 249-257

10 Boucher Y, Baxter LT, Jain RK. Interstitial pressure gradients in tissue-isolated and subcutaneous tumors: implications for therapy. Cancer Res, 1990, 50: 4478-4484

11 Hobbs SK, Monsky WL, Yuan F, et al. Regulation of transport pathways in tumor vessels: Role of tumor type and microenvironment. Proc Natl Acad Sci USA, 1998, 95: 4607-4612

12 Hashizume H, Baluk P, Morikawa S, et al. Openings between defective endothelial cells explain tumor vessel leakiness. Am J Pathol, 2000, 156: 1363-1380

13 Baluk P, Morikawa S, Haskell A, et al. Abnormalities of basement membrane on blood vessels and endothelial sprouts in tumors. Am J Pathol, 2003, 163: 1801-1815

14 Yan X, Yu Q, Guo L, et al. Positively charged combinatory drug delivery systems against multi-drug-resistant breast cancer: beyond the drug combination. ACS Appl Mater Interfaces, 2017, 9: 68046815

15 Kretzschmann VK, Fürst R. Plant-derived vascular disrupting agents: compounds, actions, and clinical trials. Phytochem Rev, 2014, 13: 191-206 
16 Thorpe PE. Vascular targeting agents as cancer therapeutics. Clinical Cancer Res, 2004, 10: 415-427

17 Pilat MJ, Lorusso PM. Vascular disrupting agents. J Cell Biochem, 2006, 99: 1021-1039

18 Dumontet C, Jordan MA. Microtubule-binding agents: a dynamic field of cancer therapeutics. Nat Rev Drug Discov, 2010, 9: 790-803

19 Baguley BC. Antivascular therapy of cancer: DMXAA. Lancet Oncology, 2003, 4: 141-148

20 Ching LM, Zwain S, Baguley BC. Relationship between tumour endothelial cell apoptosis and tumour blood flow shutdown following treatment with the antivascular agent DMXAA in mice. Br J Cancer, 2004, 90: 906-910

21 Cooney MM, van Heeckeren W, Bhakta S, et al. Drug insight: vascular disrupting agents and angiogenesis-novel approaches for drug delivery. Nat Clin Prac Oncol, 2006, 3: 682-692

22 Zhen M, Shu C, Li J, et al. A highly efficient and tumor vasculartargeting therapeutic technique with size-expansible gadofullerene nanocrystals. Sci China Mater, 2015, 58: 799-810

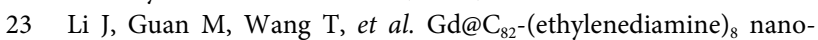
particle: a new high-efficiency water-soluble ROS scavenger. ACS Appl Mater Interfaces, 2016, 8: 25770-25776

24 Zhang Y, Shu C, Zhen M, et al. A novel bone marrow targeted gadofullerene agent protect against oxidative injury in chemotherapy. Sci China Mater, 2017, 60: 866-880

25 Chan LS, Malcontenti-Wilson C, Muralidharan V, et al. Alterations in vascular architecture and permeability following OXi4503 treatment. Anti-Cancer Drugs, 2008, 19: 17-22

26 El-Emir E, Boxer GM, Petrie IA, et al. Tumour parameters affected by combretastatin A-4 phosphate therapy in a human colorectal xenograft model in nude mice. Eur J Cancer, 2005, 41: 799-806

27 Jiang W, Huang Y, An Y, et al. Remodeling tumor vasculature to enhance delivery of intermediate-sized nanoparticles. ACS Nano, 2015, 9: 8689-8696

28 Malamas AS, Jin E, Gujrati M, et al. Dynamic contrast enhanced MRI assessing the antiangiogenic effect of silencing HIF-1a with targeted multifunctional ECO/siRNA nanoparticles. Mol Pharm, 2016, 13: 2497-2506

29 Shenoi MM, Iltis I, Choi J, et al. Nanoparticle delivered vascular disrupting agents (VDAs): use of TNF- $\alpha$ conjugated gold nanoparticles for multimodal cancer therapy. Mol Pharm, 2013, 10: 1683-1694

30 Tozer GM, Akerman S, Cross NA, et al. Blood vessel maturation and response to vascular-disrupting therapy in single vascular endothelial growth factor-A isoform-producing tumors. Cancer Res, 2008, 68: 2301-2311

31 Salmon HW, Siemann DW. Effect of the second-generation vascular disrupting agent OXi4503 on tumor vascularity. Clin Cancer Res, 2006, 12: 4090-4094

32 Kötz B, West C, Saleem A, et al. Blood flow and Vd (water): both biomarkers required for interpreting the effects of vascular targeting agents on tumor and normal tissue. Mol Cancer Therapeutics, 2009, 8: 303-309

33 Wu X, Jeong EK, Emerson L, et al. Noninvasive evaluation of antiangiogenic effect in a mouse tumor model by DCE-MRI with Gd-DTPA cystamine copolymers. Mol Pharm, 2010, 7: 41-48

34 Marzola P, Degrassi A, Calderan L, et al. Early antiangiogenic activity of SU11248 evaluated in vivo by dynamic contrast-enhanced magnetic resonance imaging in an experimental model of colon carcinoma. Clinical Cancer Res, 2005, 11: 5827-5832

35 Mikawa M, Kato H, Okumura M, et al. Paramagnetic water-soluble metallofullerenes having the highest relaxivity for MRI contrast agents. Bioconjugate Chem, 2001, 12: 510-514

36 Leunig M, Yuan F, Menger MD, et al. Angiogenesis, microvascular architecture, microhemodynamics, and interstitial fluid pressure during early growth of human adenocarcinoma LS174T in SCID mice. Cancer Res, 1992, 52: 6553-6560

37 Kohtala S, Theilmann W, Suomi T, et al. Brief isoflurane anesthesia produces prominent phosphoproteomic changes in the adult mouse hippocampus. ACS Chem Neurosci, 2016, 7: 749-756

38 Daldrup H, Shames DM, Wendland M, et al. Correlation of dynamic contrast-enhanced MR imaging with histologic tumor grade: comparison of macromolecular and small-molecular contrast media. Am J Roentgenology, 1998, 171: 941-949

39 Tofts PS, Brix G, Buckley DL, et al. Estimating kinetic parameters from dynamic contrast-enhanced t1-weighted MRI of a diffusable tracer: Standardized quantities and symbols. J Magn Reson Imag, 1999, 10: 223-232

40 Kokubo K, Shirakawa S, Kobayashi N, et al. Facile and scalable synthesis of a highly hydroxylated water-soluble fullerenol as a single nanoparticle. Nano Res, 2011, 4: 204-215

41 Zhang J, Ye Y, Chen Y, et al. $\mathrm{Gd}_{3} \mathrm{~N} @ \mathrm{C}_{84}(\mathrm{OH})_{x}$ : A new egg-shaped metallofullerene magnetic resonance imaging contrast agent. J Am Chem Soc, 2014, 136: 2630-2636

42 Zahra MA, Hollingsworth KG, Sala E, et al. Dynamic contrastenhanced MRI as a predictor of tumour response to radiotherapy. Lancet Oncology, 2007, 8: 63-74

43 Malamas AS, Jin E, Zhang Q, et al. Anti-angiogenic effects of bumetanide revealed by DCE-MRI with a biodegradable macromolecular contrast agent in a colon cancer model. Pharm Res, 2015, 32: 3029-3043

44 Pedersen M, Morkenborg J, Jensen FT, et al. In vivo measurements of relaxivities in the rat kidney cortex. J Magn Reson Imag, 2000, 12: $289-296$

Acknowledgements This work was supported by the National Natural Science Foundation of China (51472248 and 51502301), National Major Scientific Instruments and Equipments Development Project (ZDYZ2015-2), and the Key Research Program of the Chinese Academy of Sciences (QYZDJ-SSW-SLH025). We thank Zhentao Zuo for the design of the radiofrequency generator and the help by the State Key Lab of Brain \& Cognitive Sciencesin Institute of Biophysics, Chinese Academy of Sciences.

Author contributions Deng R, Zhen M and Wang C designed the devices and experiments; Li J, Yu T and Zhou Y synthesized the GFNCs; Deng R, Li X and Shu C analyzed the nature of GFNCs; Deng R and Zou $\mathrm{T}$ performed the DSFC experiments; Deng R, Wang $\mathrm{Y}$ and $\mathrm{Xu} \mathrm{H}$ performed the DCE-MRI experiments; Deng R, Wang Y and Wang C analyzed the data; Deng $\mathrm{R}$ wrote the paper with support from Wang $\mathrm{C}$ and Zhen M. All authors contributed to the general discussion.

Conflict of interest The authors declare that they have no conflict of interest.

Supplementary information online version of the paper.
Supporting data are available in the 

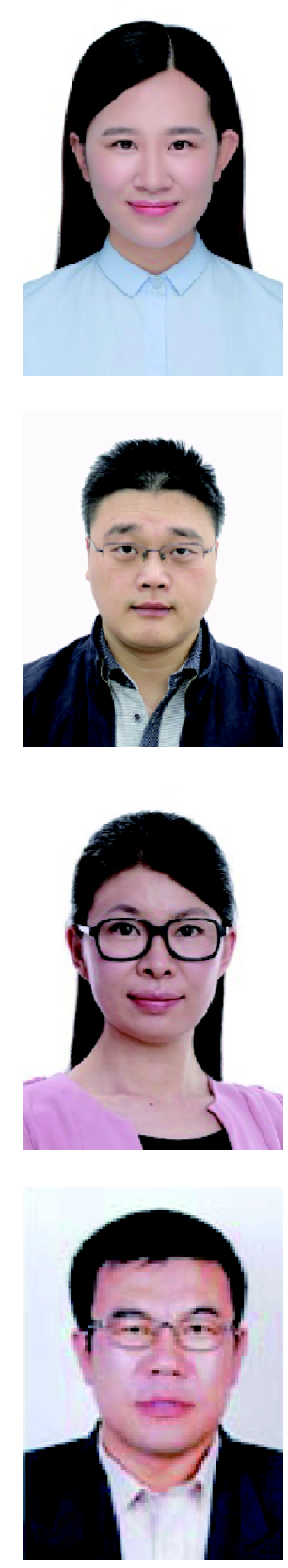

Ruijun Deng was born in 1991. She received her bachelor degree from Hebei University. She is currently pursuing her $\mathrm{PhD}$ degree in physical chemistry from the Institute of Chemistry, Chinese Academy of Sciences. Her main research includes the biomedical application of the fullerenes and gadofullerenes.
Yuqing Wang was born in 1980. He received his $\mathrm{PhD}$ degree in biomedical engineering from the University of Electronic Science and Technology in 2011. Currently, he is an engineer at the National Center for Nanoscience and Technology. His research interests refer to medical imaging processing in MR.
Mingming Zhen was born in 1987. She received her PhD degree in physical chemistry from the Institute of Chemistry, Chinese Academy of Sciences in 2014. Currently, she is an assistant professor at the Institute of Chemistry, Chinese Academy of Sciences. Her research interests include biomedical applications of fullerenes and gadofullerenes.
Chunru Wang was born in 1965. He received his PhD degree in physical chemistry from Dalian Institute of Chemistry Physics, Chinese Academy of Sciences in 1992. Currently, he is a professor at the Institute of Chemistry, Chinese Academy of Sciences. His research interests include fullerenes and endohedral fullerenes, mainly focusing on their industrialization and applications. He discovered the metal carbide fullerenes for the first time, researched on high efficiency MRI contrast agents and developed a novel tumor vascular-targeting therapy technique using gadofullerenes.

\section{射频辅助金属富勒烯纳米晶体阻断肿瘤血管的原位研究}

邓睿君 ${ }^{1,2 \dagger}$, 王昱青 ${ }^{3 \dagger}$, 甄明明 ${ }^{1,2^{*}}$, 李雪 ${ }^{1,2}$, 邹头君 ${ }^{1,2}$, 李杰 ${ }^{1,2}$, 于童 ${ }^{1,2}$, 周悦 ${ }^{1,2}$, 卢志高 ${ }^{1,2}$, 许辉 ${ }^{1}$, 舒春英 ${ }^{1,2}$, 王春儒 ${ }^{1,2^{*}}$

摘要 射频辅助金属富勒烯纳米晶体阻断肿瘤血管作为一项新兴的抗肿瘤技术, 因其高效安全的作用效果, 在癌症治疗的研究发展过程 中表现出巨大的应用前景. 本文针对该技术, 提出了对其阻断肿瘤血管的实时原位研究方法, 清晰明确地揭示了高效靶向阻断肿瘤血管的 机制. 通过建立小鼠肿瘤背部皮翼视窗模型, 实现了在治疗过程中肿瘤血管和正常血管的形态变化及血流情况的直观监测评价. 同时, 采 用临床常用的动态增强磁共振成像手段对肿瘤血管功能进行实时定量评估, 借助相关参数 $K_{\text {trans, }}$ 证明了肿瘤血管在治疗后发生了持续不 可逆的破坏. 具体表现为局部肿瘤血管出血、塌陷, 导致整个肿瘤血管网的血流停止, 切断了肿瘤组织与外界的营养交换, 进而致使肿瘤 坏死, 而正常血管并不会受到损伤. 此研究结果是对该技术高效靶向治疗肿瘤的深入研究, 有利于促进其在临床上的转化和应用. 